\title{
Coléoptères (Clavicornes, Clérides, Malacodermes, Hétéromères, Bruchides, Phytophages) d'Angola \\ par
}

Maurice PIC

Les Coléoptères qui suivent appartiennent à diverses familles. Ils ont été recueillis dans I'Angola, par le $\mathrm{D}^{\mathrm{r}} \mathrm{A}$. Monard et sont déposés au Musée d'Histoire Naturelle de La Chaux-de-Fonds (à l'exception de quelques uniques que je désirais et que leur récolteur m'a généreusement abandonnés, ce dont je le remercie cordialement).

Le $\mathrm{D}^{\mathrm{r}} \mathrm{A}$. Monard a principalement chassé dans la région du fleuve Kubango, à 500 kilomètres de la côte angolaise, et qui est peu connue au point de vue zoologique. Je dois à mon honorable correspondant, au sujet des localités, quelques précisions que je suis heureux de reproduire ci-dessous:

Lolito et Catumbela sont au bord de la mer. Ebanga, est à 220 kilomètres de là, à une altitude de $1300 \mathrm{~m}$. environ.

Caloquembé, Etonga, Lombala sont à 120 kilomètres au sud d'Ebanga et à $1600 \mathrm{~m}$. d'altitude.

Saint Amaro est à 450 kilomètres de la mer et à $1600 \mathrm{~m}$. d'altitude.

Kubango ou Vila da Ponte, Rio Mbale, Caquindo ou Cakindo, Tjitunda, Muleke, Tumbole sont dans la région du fleuve Kubango, à 1000 à $1400 \mathrm{~m}$. d'altitude.

Chimporo est un fleuve desséché, réduit à l'état de marais, à $1060 \mathrm{~m}$. d'altitude.

Les types des nouveautés sont dans ma collection et les co-types de celles récoltées en plusieurs exemplaires se trouvent au Musée d'Histoire Naturelle de La Chaux-de-Fonds.

Rev. Suisse de Zool. T. 38. 1931. 


\section{CLAVICORNES.}

\section{DERMESTIDES.}

Dermestes lardarius L. Vila da Ponte, XII.

Dermestes vulpinus F. Chimporo, XI. Vila da Ponte, XII.

\section{Scaphiditdes.}

Antongilium humerale n. sp.

Oblongo-elongatum, antice et postice attenuatum, parum nitidum, supra distincte, minute sat dense fulvo pubescens, nigrum, elytris apice testaceo marginatis et ad humeros vage rufo notatis, membris, abdomine apice pygidioque testaceis. Articulo $3 \mathrm{e}$ antennarum breve, $3 \mathrm{e}$ elongato; thorace elongato, antice attenuato, lateraliter paulo curvato et marginato, angulis posticis paulo prominulis, minute, parum dense, punctato; scutello nullo; elytris thorace non latioribus, humeris nullis, sat elongatis, lateraliter subarcuatis, postice paulo attenuatis, apice late truncatis, parum fortiter sat dense punctatis, pro parte ruguloso punctatis, stria presuturalis medio paulo impressa, antice indistincta, obliterata, epipleuris elongatis, non latis, postice attenuatis, apice indistinctis; pedibus elongatis, femoribus parum crassis, posticis infra 4 pilosis, tibiis mucronatis tarsisque gracilibus; infra corpore nitido, sat fortiter et sparse punctato. Long. $3 \mathrm{~mm}$ (coll. Pic).

Environs de Saint Amaro.

Ressemble un peu à $A$. nitidum Pic; moins brillant et plus nettement pubescent, de forme un peu plus allongée, à strie présuturale plus courte et moins enfoncée et d'ailleurs distinct, à première vue, par la coloration particulière des élytres qui sont bordés de clair avec une macule humérale roussâtre.

\section{CLÉRIDES.}

Cephaloclerus basipes Schklg.

La coloration des pattes varie un peu, les tibias étant parfois diversement roux. Espèce nouvelle pour l'Angola. Recueilli à Catumbela, au bord de la mer. 


\section{MALACODERMES.}

Apalochrus malachioides Frm. Tjitmuda.

Apalochrus sibutensis Pic et var. ․

Cette espèce, décrite de Fort Sibut, se retrouve à Angola, recueillie à Tjitunda et représentée par des o seulement variant de coloration et ayant, en majeure partie, les élytres à reflets plus ou moins dorés ou un peu cuivreux.

\section{Apalochrus monardi n. sp. ㅇ.}

Oblongus, nitidus, argenteo pubescens, metallicus, diverse viridescens, pro parte auratus, labro, epistome, palpis, antennis ad basin, pedibus abdomineque pro majore parte rufo-testaceis. Capite sat robusto, pro parte sat dense, sed diverse rugulosopunctato, supra inaequale; anternis validis, sat robustis, nigris, ad basin rufis; thorace breve et lato, supra inaequale, minute et sparse punctato; elytris thorace paulo latioribus, sat brevibus, postice paulo dilatatis, apice oblique subtruncatis, minute et dense punctatis; pedibus parum crassis, elongatis, rufo-testaceis. Long. $7-8 \mathrm{~mm}$.

Tjitunda, région du fleuve Kubango (plusieurs ex.). Très voisin de $A$. sibutensis Pic et en différant, à première vue, par les pattes de coloration entièrement claire.

Apalochrus violaceicolor Pic, Vila da Ponte, Xll.

Apalochrus ? simoni Pic ㅇ. Catumbela VII.

Apalochrus inchoatus Chp. var. Catumbela VII et VIII. (ô pattes intermédiaires largement testacées, tibias compris).

Apalochrus longicornis Chp. Catumbela VIII.

Zygia apicalis Har. Vila da Ponte, décembre; Kubango XII, Tjitunda I.

\section{LyCIDES.}

Lycus (Acantholycus) constrictus Fahr. var. integer Brg. Catumbela VIII; Chimporo XI. 
Lycus (Acantholycus) terminatus Dalm. et var. Rio Mbalé IX; Saint Amaro, Cakindo X.

Lycus sp. ․ Chimporo XI (voisin de raffrayi Bourg. probablement).

\section{HÉTÉROMÈRES.}

Notoxus rufomaculatus Pic var., Vila da Ponte XII.

Formicomus angolensis n. sp.

Satis elongatus, nitidus, niger, elytris viridescentibus, antice parum distincte albo fasciatis, antennis nigris, ad basin rufis. Capite parum robusto, sat elongato, postice attenuato, parum dense ruguloso punctato; antennis gracilibus; thorace elongato, antice dilatato et globuloso, ad basin intuberculato, fortiter parum dense punctato; elytris oblongis, antice transverse depressis, griseo pubescentibus et longe hirsutis, minute et sparse punctatis; femoribus robustis, anticis in mare fortiter sat longe dentatis, inermibus $q$, tibiis anticis ô sinuatis. Long. $4 \mathrm{~mm}$.

Catumbela, au bord de la mer (plusieurs ex.). Très voisin de $F$. spinifer Pic, en diffère par la dent fémorale plus robuste, l'avant corps à ponctuation moins dense, d'où plus brillant.

Lagria villosa F. Caluquenbe VIII; Tjitunda I.

Lagria cuprina Ths. et var. Tjitunda I.

Lagria $s p$. $\widehat{o}$ (peut-être $\widehat{\jmath}$ de Cuprina Ths.) Chimporo XI. (Cuivreux, antennes à articles médians très larges.)

Lagria sp. ㅇ (à élytres vertes, un peu cunéiformes, allongées) et 1 ô aussi probablement. Vila da Ponte XII.

Lagria flavipennis Fahr. Catumbela VIl.

Lagria (Lopholagria) crampeli Pic.

Espèce nouvelle pour l'Angola. Recueillie à la Vila da Ponte, dans la région du fleuve Kubango.

Lyprops chalceus Geb. Chimporo XI.

Zophosis sp. Planalto de Lobito VIII.

Gargilius rhodesianus Pic. Vila da Ponte XII; Ebanga XIII.

Platydema crampeli Pic et var. Ebanga VIII.

Platydema? hollmi Geb. Ebanga VIII.

Synallecula picea Sahlb. Vila da Ponte XII.

Allecula crassipes Fairm. 


\section{VÉSICANTS.}

Zonabris liquida Er. Catumbala VIII.

Ceroctis integra Har. Etonga VIII; Calaquenbé VIII; SaintAmaro 1X.

Ceroctis congoana Duv. et var. Chimporo XI.

Ceroctis trifurca Gerst. Vila da Ponte XII.

Ceroctis exclamationis Mars. Tjitunda I; Ebanga VIII; Vila da Ponte XII.

Epicauta oculata F. Vila da Ponte XII. Kubango XII (Kubango). Cyaneolytta rugipennis Makl. Caiundo X.

Cyaneolytta ? subcoriacea Makl. Cokindo X. Cyaneolytta atrocaerulea Har. Vila da Ponte.

Zonitomorpha natalensis Pic.

Le seul exemplaire récolté à Chimporo (fleuve desséché, à $1060 \mathrm{~m}$. d'altitude) a l'extrémité des élytres moins largement noire que le type originaire du Natal et les antennes ont leurs articles 4 et suivants plus larges ou plus courts, différences sexuelles sans doute.

\section{BRUCHIDES.}

Spermophagus multipunctatus Pic.

Décrit du. Sénégal, retrouvé à Angola, à Rio Mbalé région du fleuve Kubango. Cette petite espèce se reconnaît aux nombreuses mouchetures blanches ornant les élytres et à la base du pygidium ornée de pubescence jaune.

Spermophagus senegalensis Pic var. Rio Mbalé.

(Subovalaire, petit, noir assez densément et uniformément pubescent de gris, éperons des tibias roux.)

\section{PHYTOPHAGES.}

Sagra sp. Kubango XII; Vila da Ponte XII.

Lema turgida Jac. Vila da Ponte XII.

Un exemplaire rouge, assez robuste, antennes fortes, noires à base rousse, tarses noirs. 
Lema sp. (voisin de bingeri Pic) Catumbela VIII.

Petit exemplaire testacé, tête rousse, antennes foncées, à base rousse, tibias et tarses en partie rembrunis, abdomen noir à sommet clair.

\section{Lema rubricollis Klug. Catumbela VIII.}

Elytres verts, avant-corps en partie roux, tête foncée en avant, pattes bicolores, variablement foncées.

Lema rubricollis v. escaleræ Weise. Catumbela VIII.

Un exemplaire moyen, vert métallique, membres toncés.

Diapromorpha trifasciata v. scabrosa W. Saint Amaro 1X.

Diapromorpha (Peploptera) monardi n. sp.

Oblonga, postice attenuata nitida, nigra, antennis ad basin, thorace lateraliter, tibiis tarsisque rufis, elytris rufo-testaceis, ad humeros nigro maculatis, ad suturam medio et externe reducte nigro vittatis. Capite inaequale, diverse punctato, vertice sulcato; antennis brevibus, crassis, articulis intermediis latioribus; thorace parum breve et lato, antice paulo attenuato, inaequale et sparse punctato; elytris thorace non latioribus, sat elongatis, postice attenuatis, fortiter, apice fere indistincte et irregulariter punctatis; pedibus validis, tarsis dilatatis, his, infra corpore et pygidio argenteo pubescentibus. Long. $7 \mathrm{~mm}_{-} 8$.

Vila da Ponte, région du fleuve Kubango (plusieurs ex.). Peut se placer près de $P$. suturalis Clav., caractérisé par la bande latérale noire des élytres raccourcie, touchant le bord, étendue un peu avant le milieu des élytres jusque près de l'extrémité.

\section{Diapromorpha (Peploptera) minuta n. sp.}

Subparallela, antice et postice paulo attenuata, nitidanigra, antennis ad basin rufis, elytris antice reducte et late testaceo fasciatis (calus humeralis niger) et apice testaceo-marginatis. Capite fortiter punctato, inter oculos impresso; antennis parum brevibus, latis; thorace breve et lato, antice paulo attenuato, lateraliter minute marginato, fortiter et sparse punctato; elytris thorace parum distincte latioribus, sat elongatis, lateraliter subsinuatis, postice attenuatis, minute marginatis, irregulariter pro parte fortiter punctatis, punctis apice obliteratis, margina 
apicalis nigra; infra corpore parum pubescente; pedibus sat validis. Long. $4 \mathrm{~mm}$.

Vila da Ponte (plusieurs ex.). Par sa petite taille cette espèce peut prendre place près de pusilla Lef. mais elle est d'une toute autre coloration.

Clytra wahlbergi Lac. Vila da Ponte XII.

Melitonoma? bomaensis Jac. Catumbela VII.

A 5 macules noires (1, 2, 2 en fascie) sur les élytres.

Melitonoma sp. Tjitunda I.

Prothorax maculé de foncé, élytres à 6 macules en lignes 2, 2, 2 - foncées, humérale et postérieure latérale allongées).

Coptocephala (Anisognatha) hyacinthina Har. var.

Recueilli en plusieurs exemplaires, de nuances variées, au bord de la mer, à Catumbela, mais aucun individu ne se rapporte à la nuance typique à élytres entièrement d'un vert métallique.

Deux variétés nouvelles, à élytres en partie marquées de jaune, se reconnaitront ainsi qu'il suit:

Elytres métalliques avec une bordure testacée latérale, plus ou moins étendue; nuance citée et non nommée par Lacordaire (var. lateralis, plusieurs ex.).

Elytres à bordure claire, prolongée en s'élargissant sur la suture à l'extrémité, en outre ornés chacun, près de la base, de 2 petites macules testacées (var. basinotatus, un ex. in coll. Pic).

Je suis disposé à croire que Anisognatha discors Er., recueilli dans la même localité, est une autre nuance d'une unique espèce et dans ce cas, discors Er. étant décrit avant hyacinthina Er., c'est ce nom qui devrait être adopté comme spécifique.

Coptocephala (Anisognatha) discors Er. Catumbela VIII.

(Elytres un peu allongés, à dessins verts: 1 macule, 1 fascie réduite.) Coptocephala discors var. Tjitunda I.

(Variété du précédent, élytres moins allongées à 1, 1,2 macules métalliques, macule interne postérieure parfois effacée.

Coptocephala sp. Saint Amaro XI.

(Elytres testacés à bande suturale et bande humérale effacées en arrière vertes.) 
Cryptocephalus 4-undulatus var. potensis, var. nov.

Robuste, brillant, testacé jaunâtre, écusson poitrine en partie, extrêmité des antennes et tête noires, celle-ci bilinéolée de clair sur son milieu; prothorax jaune, orné de 2 macules basales noires isolées et, au milieu de chaque côté, de 2 macules discales, jointes par un trait; élytres à rangées de points fins, jaunes, ornés sur chacun d'une courte bande basale externe échancrée, d'une courte bande suturale médiane sinuée et d'une fascie anteapicale complète et sinuée noires.

Un exemplaire, Vila da Ponte III (in coll. Pic), à dessous peu pubescent, distinct de la forme type par les dessins noirs particuliers du prothorax et les dessins fasciés des élytres un peu différents.

Cryptocephalus bilunulatus Pic. Muleke I et Chimporo XI.

(Prothorax à 4 macules noires.)

Cryptocephalus 10 notatus Sufr. var. Vila da Ponte XII.

(Prothorax à 4 macules noires, élytres immaculés, jaunes.) Cryptocephalus lowei v. plebejus Rein. Tjitunda I.

\section{Melixanthus monardi n. sp.}

Subovatus, convexus, nitidus, rufus, antennis apice, scutello, infra corpore, pygidio, femoribus posticis ad basin tarsisque pro parte, nigris, elytris ad basin et ante apicem viride-fasciatis, fascia prima lateraliter et infra humeros prolongata, fascia postica sinuata, sutura inter fascias nigro-viridescens. Antennis apice sat validis; capite viridescente, fortiter punctato, oculis valde distantibus; thorace breve, antice attenuato, minute et sparse punctato, elytris latis et brevibus, postice attenuatis, lineatopunctatis; pygidio forticr punctato. Long. $3 \mathrm{~mm}$. Tjitunda I (deux exemplaires).

Cette petite espèce est caractérisée par les bandes verdâtres des élytres et se distingue ainsi facilement des autres espèces du genre.

Melixanthus conradti Rein. Vila da Ponte XII.

Chlamys sp. Rio Mbalé X.

Colasposoma sp. Tjitunda I.

Malegia atritarsis $\mathrm{n}$. sp.

Oblonga, parum convexa, nitida, uniformiter non dense griseo pubescens, parum fortiter et dense punctata, nigro olivacea, supra 
olivaceosubaenea, antennis nigris, ad basin rufis, pedibus rufis, tarsis nigris. Capite antice triangulariter inciso, oculis prominulis; antennis sat gracilibus et elongatis; thorace parum breve, sat lato, lateraliter subarcuato; elytris thorace sat latioribus, parum elongatis, apice attenuatis, irregulariter parum dense punctatis; pedibus parum validis. Long. $3 \mathrm{~mm}$.

Cokindo, région du fleuve Kubango (2 exemplaires). Ressemble à $M$. letourneuxi Lef., en diffère par le dessus du corps un peu convexe, la ponctuation moins forte et plus dense du dessus du corps et les tarses foncées.

Dorcathispa sp. (ou bellicosa Guer. ?) Saint Amaro XI.

Dorcathispa alternata W. Catumbela VII.

Pseudhispella militaris W. Saint Amaro IX. 


\section{$2 \mathrm{BHL}$ Biodiversity Heritage Library}

Pic, Maurice. 1931. "Coléoptères (Clavicornes, Clérides, Malacodermes, Hétéromères, Bruchides, Phytophages) d'Angola." Revue suisse de zoologie 38, 419-427. https://doi.org/10.5962/bhl.part.117647.

View This Item Online: https://www.biodiversitylibrary.org/item/148559

DOI: https://doi.org/10.5962/bhl.part.117647

Permalink: https://www.biodiversitylibrary.org/partpdf/117647

\section{Holding Institution}

American Museum of Natural History Library

\section{Sponsored by}

BHL-SIL-FEDLINK

\section{Copyright \& Reuse}

Copyright Status: In copyright. Digitized with the permission of the rights holder.

Rights Holder: Muséum d'histoire naturelle - Ville de Genève

This document was created from content at the Biodiversity Heritage Library, the world's largest open access digital library for biodiversity literature and archives. Visit BHL at https://www.biodiversitylibrary.org. 